\title{
Review of Energy Efficient Techniques of IoT
}

\author{
Ritesh Awasthi \\ Research Scholar, M.tech Pursuing, CSE \\ GGSCMT, Kharar \\ Punjab,India \\ riteshavasthi27a@gmail.com
}

\author{
Navneet Kaur \\ A.P (CSE), \\ GGSCMT, Kharar \\ Punjab,India \\ kaur.navneet26@gmail.com
}

\begin{abstract}
The network across which the information is sensed by the sensor devices and then forwarded to the sink is known as Internet of Things (IoT). Even though this system is deployed in several applications, there are certain issues faced in it due to its dynamic nature. The internet of things is derived from the wireless sensor networks. The sensor nodes which are deployed to sense environmental conditions are very small in size and also deployed on the far places due to which energy consumption is the major issue of internet of things. This research work related to reduce energy consumption of the network so that lifetime can be improved. In the existing system the approach of multilevel clustering is used for the data aggregation to base station. In the approach of multilevel clustering, the whole network is divided into clusters and cluster heads are selected in each cluster. The energy efficient techniques of internet of things are reviewed and analyzed in terms of certain parameters.
\end{abstract}

Keywords- IoT, Clustering, Energy Efficient, WSN.

$* * * * *$

\section{Introduction}

Internet of things is the collection of things, especially the objects being used in daily routine, which can be readable, recognizable, locatable, and addressable, by using sensing devices. These things are controlled by internet services with respect to their communication means. These objects not only includes the electronic devices but it includes the products having higher technical development like vehicles and other appliances but such things which are not electronic are also include in this, for e.g. food, clothing, chair, animal, trees and water and many more. Internet of things is new invention in the field of technology. They have the ability to access through all the information which has been collected from other things or they can also integrate this information into smaller pieces. This information is associated with the cloud computing and having unlimited addressing tendency.

\section{Characteristics of IoT}

Following are the important characteristic properties of IoT:

1) Interconnectivity: According to the IoT, everything is connected each other through global communication and communication infrastructure.

2) Things-related services: The IoT has a capacity to provide things related services in the constraints of services like protection of data services and synchronized consistent services in between the physical and virtual [1] things. It provides the things-related services in the constraints of things, both in the technological as well as in the physical world.
3) Heterogeneity: Depending upon the various hardware platforms and networks which exist in applications, different kinds of devices are deployed in IoT. Various networks are used by these devices as intermediates for interacting with other service platforms.

4) Dynamic changes: The dynamic changes either in services or in location and speed are provided through this network. Thus, it is possible to change the number of appliances dynamically.

5) Enormous scale: In comparison to the devices connected to internet, there are higher numbers of devices that require management and communication services with other devices.

Safety: With the advancement of benefits of IoT, one mustnot forget about the safety measures of it. This should be properly designed for the creators and recipients keeping in mind the safety issues. It includes the protection of

6) Safety: With the advancement of benefits of IoT, one must not forget about the safety measures of it. This should be properly designed for the creators and recipients keeping in mind the safety issues. It includes the protection of personal data and the protection of physical well-being. It also secures the endpoints, the network, and the data stored in that network.

7) Connectivity: It has network accessibility and compatibility. Accessibility provides proper access to the network however, the ability to consume and create data is provided through compatibility. 


\section{Data Aggregation}

The data aggregation is the approach through which data is collected from the sensor nodes and passed to base station with least redundancy. It requires sensor data which is extracted from the sensor node and then collects the data with the help of some aggregation techniques. This collected data is further forwarded to the sink node by choosing and selecting the appropriate and relevant data. The elimination and removal of all the irrelevant and redundant data from the network and then improving the lifetime of energy are the important tasks of this approach. Data transmission is performed in multi-hop manner in which every node transfers its data to its nearby node. So, the nearby node will easily sense the data [6]. In order to overcome this limitation, another approach was introduced in which every node will transfer its data to the cluster-head which will perform the function of data aggregation and finally send the data to the sink for further processing. This will cause wastage of energy and in wireless sensor network the cluster head will not work much longer. Due to this all the processing has to perform again which will waste time as well as energy.

\section{Techniques used in Data Aggregation}

Several data aggregation techniques have been developed over the years.

Centralized Approach: It is an address centralizedtechnique in which every node will sends the data to the central node through the shortest possible path. Sensor node is the powerful node and act as leader of all the nodes and is responsible to transmit data packets forward for the further processing. This will aggregate the data which is being stored in the network. Every node present in between the path has to send their data packets to the sensor node. Therefore, huge amount of data and messages are transmitted for a query.

Tree-Based Approach: In this type of approach theaggregation will takes place in the form of tree, which can be a reduced spanning tree, having roots which behave [7] as sink and leaves will act as a source node. Every node consists of parent node which is used to forward its entire data for further processing. The data will start flowing from the leaves through sink and then aggregation will takes place at the parent node.

Cluster-Based Approach: Clusters are created bydividing the complete network using this approach. A cluster head is chosen individually for each cluster, amongst all the nodes. The data is collected and aggregated from other nodes in the cluster by this cluster head due to which it is also known as aggregator. In order to perform the final processing, this aggregated data is forwarded to the sink.
In-Network Aggregation: In this approach,information is gathered and routed by the multi-hop network. This has the ability to reduce the consumption of resource which continuously increasing the lifetime of the network. It is divided into two more approaches called the approach that minimize the size and the approaches that don't minimize the size. The data packets from the nodes are combined and compressed within the size reduction approach. Further, the packet length that is to be transmitted and forwarded to sink is reduced using these nodes. The mechanism of collaboration of all the data packets received from all the neighboring nodes and processing them without the value of data is the approach that is without size reduction.

\section{Literature Review}

Emma Fitzgerald [8] presented a study which was related to the different algorithms and formulations of mixed-inter programming. The issues of energy-optimal routing and multiple-sink aggregation within IoT edge networks were solved by this approach. Due to the least and highest utilization of energy, it is important to consider optimization of network. In case of pure aggregation, the throughput optimal scheduling of transmissions is done using physical inference model. The two used cases and the time required to solve them are compared by the numerical study performed here. There are around 10 to 40 nodes within the topologies of network. However, for less than $15 \%$ of the total energy being used in all test cases, good results are achieved through aggregation. Substantial amount of energy is saved through this approach. It is seen that by utilizing the direct and shortest-path flows from sensors to actuators around 13 times higher energy is utilized by the 40-node networks.

Ali Kadhum Idrees et al. [9] proposed a novel approach through which the lifetime of PWSNs which is named as DiDAMoK. Within the sensor nodes, this lifetime is distributed uniformly and it works in a periodic manner.

There are three stages amongst which these periods are setup. All the collected and saved data within the sensor nodes is read by the sensor node in the initial stage. These readings are then transformed into a set and there are dynamic clusters generated which relay on the nature of aggregated readings. For transmitting the cluster to sink, one reading is chosen as the representative reading in the final stage.

Heng Chuan Tan et al. [10] proposed a novel protocol through which the integrity and authenticity to data was provided known as end-to-end integrity protocol. The elliptic curve based chameleon hashing was applied in this proposed protocol. In order to perform verifications, the gathered chameleon hash values are generated and then forwarded to the MDMS. For making sure that the resulted chameleon hash value and previous has value are equal to each other, a trapdoor is used which calculates the assured value and 
forwards it to MDMS. The integrity and authenticity of dataset is achieved by the proposed approach as per the comparative analysis performed here. Also, the computational cost is reduced by this proposed mechanism. An informal security analysis is performed in this paper through which the security measures of proposed protocol are defined.

Shilpa Rao et al. [11] presented a study in which the data is transmitted by the IoT devices to the base station using the User Equipments (UEs), otherwise known as uplink transmission. In order to perform further processing, the data is transmitted from the base station to the cloud. From the IoT devices, the data is forwarded to the user equipments. This data, along with the uplink data is aggregated to the base station in the next step. Very minimal amount of energy is required when using the user equipments in IoT as per this study. The cases where IoT devices are associated with UEs using either the random, greedy or fixed approaches are studied here. For each of these three approaches, the end-toend outage probability at the devices is evaluated. Least outage probability at the IoT devices is achieved as per the conclusion achieved while applying the proposed technique. Lijun Dong et al. [12] proposed a novel technique which acted as a solution to the problems arising in Information Centric Networking (ICN). The average number of notifications being received in single and multiple producer scenarios are minimized using this mechanism. The subscription and redundant notification messages are transmitted across the network using the least possible bandwidth through this research. The subscription condition is gathered successfully and then allocated into disjoint sets of proper transmission using the proposed technique. Thus, the duplicate notifications which carry the similar updated information from multiple producers are removed by this proposed technique.

Table 1: Table of Comparison

\begin{tabular}{|c|c|c|c|}
\hline Author & Year & Description & Outcome \\
\hline Emma Fitzgerald & & $\begin{array}{l}\text { The issues of energy-optimal } \\
\text { routing and multiple-sink } \\
\text { aggregation within IoT edge } \\
\text { networks were solved by this } \\
\text { approach. Due to the least and } \\
\text { highest utilization of energy, it } \\
\text { is important to consider } \\
\text { optimization of network }\end{array}$ & $\begin{array}{l}\text { There are around } 10 \text { to } 40 \\
\text { nodes within the } \\
\text { topologies of network. } \\
\text { However, for less than } \\
15 \% \text { of the total energy } \\
\text { being used in all test cases, } \\
\text { good results are achieved } \\
\text { through aggregation. }\end{array}$ \\
\hline Ali Kadhum Idrees & & $\begin{array}{l}\text { There are three stages amongst } \\
\text { which these periods are setup. } \\
\text { All the collected and saved } \\
\text { data within the sensor nodes is } \\
\text { read by the sensor node in the } \\
\text { initial stage. These readings are } \\
\text { then transformed into a set and } \\
\text { there are dynamic clusters } \\
\text { generated which relay on the } \\
\text { nature of aggregated readings. }\end{array}$ & $\begin{array}{l}\text { For transmitting the cluster } \\
\text { to sink, one reading is } \\
\text { chosen as the } \\
\text { representative reading in } \\
\text { the final stage. }\end{array}$ \\
\hline Heng Chuan Tan et al & & $\begin{array}{l}\text { The elliptic curve based } \\
\text { chameleon hashing was } \\
\text { applied in this proposed } \\
\text { protocol. In order to perform } \\
\text { verifications, the gathered } \\
\text { chameleon hash values are } \\
\text { generated and then forwarded } \\
\text { to the MDMS. For making sure } \\
\text { that the resulted chameleon } \\
\text { hash value and previous has } \\
\text { value are equal to each other, a } \\
\text { trapdoor is used which }\end{array}$ & $\begin{array}{l}\text { An informal security } \\
\text { analysis is performed in } \\
\text { this paper through which } \\
\text { the security measures of } \\
\text { proposed protocol are } \\
\text { defined. }\end{array}$ \\
\hline
\end{tabular}




\begin{tabular}{|c|c|c|}
\hline & $\begin{array}{l}\text { calculates the assured value } \\
\text { and forwards it to MDMS. }\end{array}$ & \\
\hline Shilpa Rao et al & $\begin{array}{l}\text { In order to perform further } \\
\text { processing, the data is } \\
\text { transmitted from the base } \\
\text { station to the cloud. From the } \\
\text { IoT devices, the data is } \\
\text { forwarded to the user } \\
\text { equipments. This data, along } \\
\text { with the uplink data is } \\
\text { aggregated to the base station } \\
\text { in the next step. Very minimal } \\
\text { amount of energy is required } \\
\text { when using the user } \\
\text { equipments in IoT as per this } \\
\text { study. }\end{array}$ & $\begin{array}{l}\text { Least outage probability at } \\
\text { the IoT devices is achieved } \\
\text { as per the conclusion } \\
\text { achieved while applying } \\
\text { the proposed technique. }\end{array}$ \\
\hline Lijun Dong et al & $\begin{array}{l}\text { The average number of } \\
\text { notifications being received in } \\
\text { single and multiple producer } \\
\text { scenarios are minimized using } \\
\text { this mechanism. }\end{array}$ & $\begin{array}{l}\text { Thus, the duplicate } \\
\text { notifications which carry } \\
\text { the similar updated } \\
\text { information from multiple } \\
\text { producers are removed by } \\
\text { this proposed technique. }\end{array}$ \\
\hline Sabin Bhandari et al. & $\begin{array}{l}\text { The packets which are being } \\
\text { received are assigned with } \\
\text { different MAC layer attributes } \\
\text { by the proposed prioritized } \\
\text { channel access approach. } \\
\text { Further, before sensing the } \\
\text { aggregated data to cloud, the } \\
\text { separate low-priority and high- } \\
\text { priority queues are utilized }\end{array}$ & $\begin{array}{l}\text { It is seen through the } \\
\text { comparative analysis that } \\
\text { the system latency and } \\
\text { reliability are improved by } \\
\text { applying the proposed } \\
\text { technique. }\end{array}$ \\
\hline
\end{tabular}

\section{Conclusion}

In this paper, it is concluded that the internet of things is the popular technology in which devices can sense the information and transmit it to base station. The major problems being faced in IoT are energy consumption and data aggregation because of the dynamic nature of the networks. The lifetime of WSNs can be enhanced by applying the clustering approach. Depending upon the distance and energy of nodes, cluster heads are chosen for each cluster. The data that is collected from the nodes is forwarded to the sink by these cluster heads. In future, novel approach will designed which improve lifetime of IoT.

\section{References}

[1]. Dr. Ovidiu Vermesan SINTEF, Norway, Dr. Peter FriessEU, Belgium, "Internet of Things: Converging Technologies for Smart Environments and Integrated Ecosystems", river publishers' series in communications, 2013.

[2]. Dr. Ovidiu Vermesan SINTEF, Norway, Dr. Peter FriessEU, Belgium, "Internet of Things-From Research and Innovation to Market Deployment", River publishers' series in communications, 2014.

[3]. G. Kortuem, F. Kawsar, V. Sundramoorthy, and D. Fitton, "Smart objects as building blocks for the internet of things," IEEE Internet Computing, Volume: 14 , Issue: 1, pp. 30-37, 2010.

[4]. Martin Serrano, Insight Centre for Data Analytics, Ireland ,Omar Elloumi, Alcatel Lucent, France, Paul Murdock, Landis + Gyr, Switzerland, "Alliance for Internet of Things Innovation", Semantic Interoperability”, Release 2.0, AIOTI WG03 - loT Standardisation, 2015.

[5]. C. Liu, K. Wu, and J. Pei, "An energy-efficient data collection framework for wireless sensor networks by exploiting spatiotemporal correlation," IEEE Trans. Parallel and Distributed Syst., vol. 18, no. 7, pp. 1010-1023, 2007.

[6]. V. Stankovic, L. Stankovi ' c, S. Wang, and S. Cheng, "Distributed ' compression for condition monitoring of wind farms," IEEE Trans. Sustainable Energy, vol. 4, no. 1, pp. 174-181, 2013.

[7]. N. Deligiannis, E. Zimos, D. Ofrim, Y. Andreopoulos, and A. Munteanu, "Distributed joint source-channel coding with copulafunction-based correlation modeling for wireless 
sensors measuring temperature," IEEE Sensor J., vol. 15, no. 8, pp. 4496-4507, 2015.

[8]. Emma Fitzgerald, "Energy-Optimal Data Aggregation and Dissemination for the Internet of Things," IEEE Internet of Things Journal, Volume: 5, Issue: 2, 2018.

[9]. Ali Kadhum Idreesa, Wathiq Laftah Al-Yaseenb, Mohamad Abou Taamc and Oussama Zahwe, "Distributed Data Aggregation based Modified K-means Technique for Energy Conservation in Periodic Wireless Sensor Networks", 2018 IEEE Middle East and North Africa Communications Conference (MENACOMM)

[10].Heng Chuan Tan, Kelvin Lim, Sye Loong Keoh, Zhaohui Tang, David Leong, Chin Sean Sum, "Chameleon: A Blind Double Trapdoor Hash function for Securing AMI Data Aggregation", IEEE 4th World Forum on Internet of Things, 2018.

[11].Shilpa Rao and Rajeev Shorey, "Efficient Device-to-Device Association and Data Aggregation in Industrial IoT Systems", 9th International Conference on Communication Systems and Networks (COMSNETS), 2017.

[12].Lijun Dong and Guoqiang Wang, "INADS: In-Network Aggregation and Distribution of IoT Data Subscription in ICN", IEEE International Conference on Multimedia \& Expo Workshops (ICMEW), 2017.

[13].Sabin Bhandari, Shree Krishna Sharma, Xianbin Wang, "Latency Minimization in Wireless IoT Using Prioritized Channel Access and Data Aggregation”, GLOBECOM 2017 - 2017 IEEE Global Communications Conference 